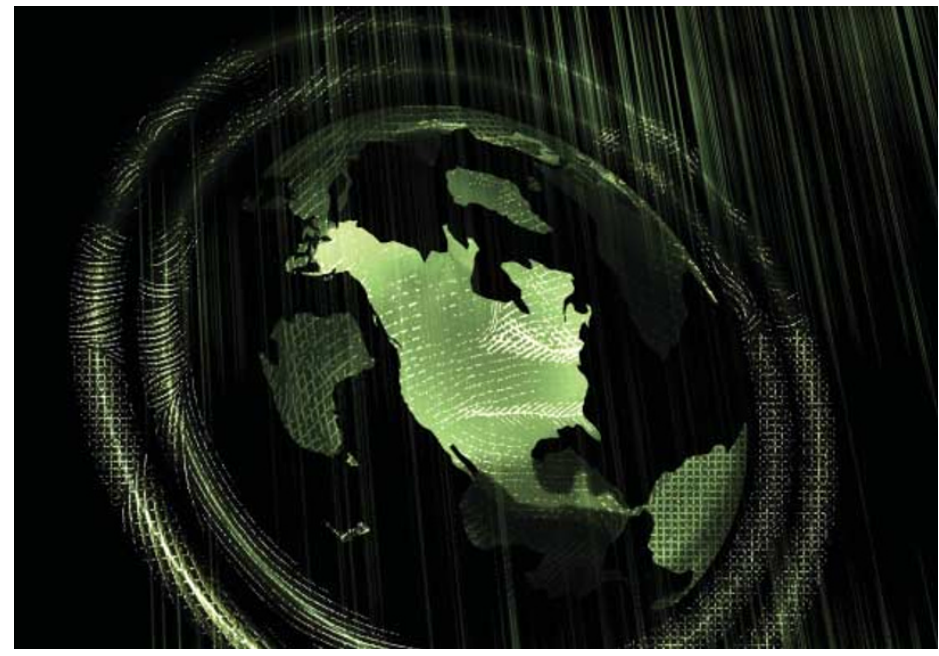

\section{Cholesterol goes global}

Lipid rafts are involved in regulating important cellular processes — such as signal transduction - and cholesterol has a central role in determining the behaviour of these specialized membrane domains. Depletion of cholesterol disrupts lipid rafts and affects the processes that occur at these platforms, but the global effects of cholesterol depletion have been overlooked. Now, Michael Edidin and colleagues report that depletion of membrane cholesterol causes changes in the actin cytoskeleton, which result in global changes in plasma-membrane function.

The authors grew human fibroblasts and lymphoblasts in a low density lipoprotein (LDL)-deficient medium to reduce cholesterol levels and then measured the membrane mobility of human leukocyte antigen 1 (HLA) molecules on the plasma membrane. Lateral diffusion of the HLA molecules was reduced when cholesterol levels were low and, after LDL was added to the medium, it took more than $24 \mathrm{~h}$ to restore mobility to the same level as the controls. By contrast, inhibiting actin polymerization - by adding cytochalasin D to the cholesterol-depleted cells - rapidly restored mobility, so changes in the actin cytoskeleton must accompany the reduced cholesterol levels.

To investigate this further, the authors used laser trapping to capture HLA molecules tagged with 40-nm gold beads. They showed that $50 \%$ of the tagged HLA molecules on cholesterol-depleted cells underwent elastic recoil, which is a rare event in normal cells. Furthermore, it was easier for the tagged HLA molecules to be pulled perpendicular to the cell membrane in the cholesterol-depleted cells, which indicated that HLA molecules are confined by the elastic membrane cytoskeleton rather than being anchored to it. Moreover, cholesterol depletion altered the activity of actin and actin-modifying proteins, which confirms that cholesterol stabilizes the actin cytoskeleton.

As these observations are similar to those seen when plasma-membrane phosphatidylinositol-4,5-bisphosphate (PtdIns $\left.(4,5) \mathrm{P}_{2}\right)$ is lost or redistributed, the authors investigated PtdIns $(4,5) \mathrm{P}_{2}$ distribution in cholesterol-depleted cells. Plasma-membrane PtdIns(4,5)P levels — measured using a green fluorescent protein (GFP)-tagged plekstrin-homology domain (PH) from phospholipase-C- $\delta$ - were reduced after cholesterol depletion. In cells expressing the $\mathrm{PH}$ domain, which binds and sequesters PtdIns $(4,5) \mathrm{P}_{2}$, the lateral diffusion of HLA molecules and their elastic recoil after laser trapping were similar to the diffusion and recoil seen in cholesterol-depleted tion regulates the actin cytoskeleton in a similar manner to cholesterol depletion.

This is therefore the first study that shows how cholesterol levels can globally alter the actin cytoskeleton by affecting the organization of PtdIns $(4,5) \mathrm{P}_{2}$. It also clearly demonstrates that cellular functions influenced by membrane-cholesterol levels can be independent of lipid rafts.

Emma Croager

(0) References and links ORIGINAL RESEARCH PAPER Kwik, J. et al. Membrane cholesterol, lateral mobility, and the phosphatidylinositol 4,5-bisphosphate-dependent organization of cell actin. Proc. Nat/ Acad. Sci. USA 100, 13964-13969 (2003)

WEB SITE

Michael Edidin's laboratory: http://www.bio.jhu fibroblasts. So, PtdIns $(4,5) \mathrm{P}_{2}$ depleedu/Directory/Faculty/Edidin/Default.htm
H I G H L I G H T S

\section{STRUCTURE WATCH}

\section{Trapped!}

Small $\mathrm{G}$ proteins (for example, Arfs) are activated when guanine nucleotide exchange factors (GEFs; for example, ARNO) catalyse the exchange of GDP for GTP. This process involves G-proteinGDP-GEF intermediates, but, owing to their transient nature, the structures of these intermediates have remained a mystery. Now, though, Cherfils and colleagues provide new insights in Nature.

Arf proteins regulate membrane trafficking in eukaryotic cells, and they couple their GDP/GTP exchange to cytosol-membrane translocation. Transient GDP-bound intermediates of these $G$ proteins can be trapped either by the natural inhibitor brefeldin A, or by a mutation in the catalytic Sec7 domain of their GEFs (E156K in ARNO). The authors therefore determined the crystal structures of these intermediates, and found that they seem to represent two actual intermediates of the exchange reaction that is, a G-protein-GDP-GEF docking intermediate and a preGDP-dissociation intermediate. The structures indicate that the Sec7 domain catalyses the membrane recruitment of Arf and the dissociation of GDP from Arf as separate, sequential reactions, and that these events involve the sequential rotation of the Arf-GDP core towards the Sec7 catalytic site. The two structures represent unproductive G-protein-GEF complexes and have highlighted "...how efficient and selective inhibition of a cellular process in vivo can be achieved by a drug that targets a transient protein-protein intermediate of a regulatory reaction and traps it in a non-productive conformation".

REFERENCE Renault, L. et al. Structural snapshots of the mechanism and inhibition of a guanine nucleotide exchange factor. Nature 426, 525-530 (2003)

\section{A refined model}

The Sec61 (eukaryotes) or SecY (eubacteria and archaea) complex is a protein-conducting channel that allows polypeptides to cross, or integrate into, membranes. It's a heterotrimeric complex with $\alpha$-, $\beta$ - and $\gamma$-subunits, and the $\alpha$-subunit forms the pore of the channel. The channel is passive and it associates with other components that provide the driving force for translocation. So far, only relatively low-resolution structures of this complex have been available, but now, in Nature, Rapoport and colleagues describe the 3.2-A resolution crystal structure of the SecY complex from Methanococcus jannaschii. This structure, combined with published data, has allowed the authors to propose models for the various steps of protein translocation.

The structure indicates that a single copy of SecY functions as a channel, despite the fact that this complex forms oligomers during translocation. A cytoplasmic funnel that leads into the channel is plugged by a short helix, and the authors propose that displacement of this plug opens the channel. The channel is hourglass shaped with a 'pore ring' of hydrophobic residues at its narrowest point. These residues might form a seal around the translocating polypeptide to block the passage of other molecules. The structure also indicates how the complex interacts with the polypeptide's signal sequence and the components that provide the driving force. Furthermore, it shows how the transmembrane segments of nascent membrane proteins might move laterally into lipid, and has provided the basis for future experimental testing.

REFERENCE van den Berg, B. et al. X-ray structure of a protein-conducting channel. Nature 3 Dec 2003 (doi:10.1038/nature02218) 